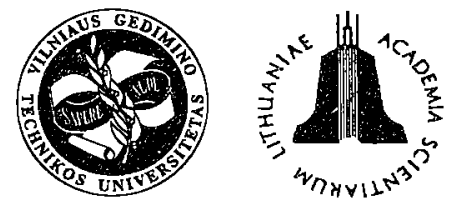

ISSN 1648-4142 TRANSPORT

http:/www.vtu.itenglish/editions

\title{
THE INVESTIGATION OF DYNAMICS OF MISALIGNED UNITS IN VEHICLE TRANSMISSION
}

\author{
Vytautas Turla, Igor Iljin \\ Department of Printing Machines, Vilnius Gediminas Technical University, \\ J. Basanavičaus g. 28,LT-2009 Vilnius, Lithuania, tel. (+370)5 2603689.E-mail: pgkatedra@me.vtu.lt
}

Received 200205 10; accepted 20021021

\begin{abstract}
In the article the problems related to the dynamics of a mechanic system on misalignment of shafts in radial direction are presented. The object of the investigation is a two-shaft system connected with an elastic centrifugal ring coupling.

Using equations of static equilibrium it was found that an internal moment of resistance to rotation appears in the coupling connecting the radially misaligned shafts. Using Dalamber's principle for the rotational movement the differential equation that describes the rotation of the second shaft has been developed. It was shown that after the performance of the corresponding actions and the introduction of a new variable the said equation is transformed in to an equation which character virtually coincides with the equation describing free oscillations of a mathematical pendulum.

Because the value of misalignment of shafts in the radial direction is small in comparison with other parameters, a small parameter method was used for the solution of this equation. The found solutions show that rotational vibrations with the double frequency of rotational movement are excited in the misaligned mechanical system with an elastic centrifugal ring coupling.

The restrictions of the application of a small parameters method have been explored and the limits of its application have been found.

Keywords: vehicle transmission, elastic centrifugal ring coupling, misaligned shafts, rotational vibrations, small parameter method.
\end{abstract}

\section{Introduction}

In vehicles with rear driving wheels a rotational movement is transferred from the engine to them via a number of mechanical equipments. On driving the body with the engine vibrates in the direction perpendicular to the longitudinal axis of the transmission and this causes radial misalignments of axes of shafts in units of the transmission. On the rotation of shafts of the units this causes variable loads on their fittings, so the rotational movement becomes uneven. This phenomenon causes sensible vibrations in the vehicle as well as the reduction of the service time of units and assemblies for periodically varying loads. The problem of the reduction of vibrations is discussed in many works, for example [1-3].

\section{The Object of the Investigation}

One of the methods of the reduction of unevenness of a rotational movement is based on the usage of elastic centrifugal ring couplings in the cardan drive. It was already described in [4]. Herein, we'll discuss the dynamic processes in such drive when shafts being misligned in radial direction are connected using the above-mentioned couplings.
The construction of the simplest elastic centrifugal ring coupling is presented in Fig 1.

The coupling consists of the driving half-coupling 1 and the driven half-coupling 2 . The terminals 3 and 4 of the half-couplings are connected with the elastic steel ring 5 , usually made of a wire wound into a circle. When the driving half-coupling is loaded with the moment of rotation a slight angular shifting of the half-couplings in respect of each other takes place on their rotation because

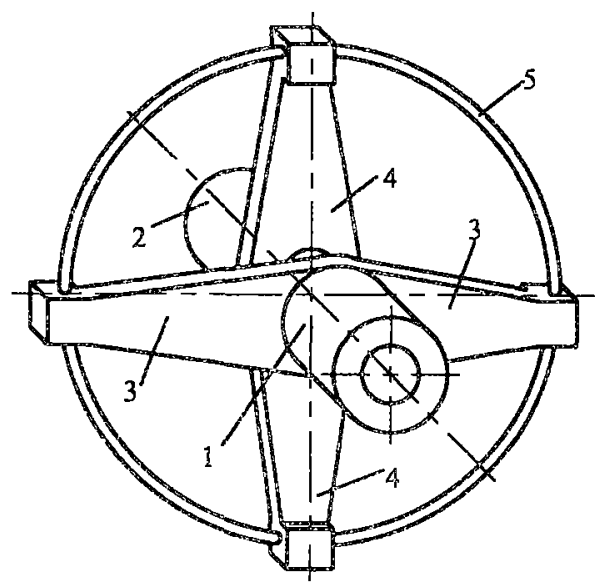

Fig 1. The construction of the simplest elastic centrifugal ring coupling 
of elastic deformations of the ring 5 and the shape of the ring becomes similar to an ellipsis. On the rotation of the system the forces of elasticity of the ring and the centrifugal forces of its distributed mass seek for the restoration of the initial round shape of the ring and simultaneously reduce the deformation of the coupling. So links of two types (elastic and centrifugal) participate in the transmission of a rotational movement [5].

\section{Connection of Shafts Misligned in Radial Direction}

The above-described situation is characteristic for the connection of ideally aligned shafts. In most cases the axes of the shafts do not coincide, for example, because of vibrations of the body in respect of driving wheels that appear in the transmission of the vehicle on driving.

In the case of radial misalignment of connected shafts the shape of the deformed elastic ring will be another (Fig 2); the reactions $P_{\mathrm{x}}$ and $P_{\mathrm{y}}$ appear in the fitting points of the terminals of the half-couplings.

In this case potential energy of the deformed elastic ring is found from the following expression:

$$
\Pi=\Sigma_{n} \int_{0}^{\frac{2 \pi}{n}} \frac{M_{l} R d \varphi}{2 E I}+\Sigma \int_{n}^{\frac{2 \pi}{n}} \frac{N_{u} R d \varphi}{2 E F},
$$

where $M_{1}$ - the moment of bending in the ring; $N_{\mathrm{a}}$ - the axial force in it; $R$ - the radius of the bend of the ring; $E I$, $E F$ - the stiffness of the ring for bending and lengthening; $\varphi$ - an angular coordinate; $n$ - the number of ring segments between the terminals of half-couplings.

Taking into consideration that

$$
\begin{aligned}
& \frac{\partial \Pi}{\partial P_{x}}=e \cos \gamma, \\
& \frac{\partial \Pi}{\partial P_{y}}=e \sin \gamma
\end{aligned}
$$

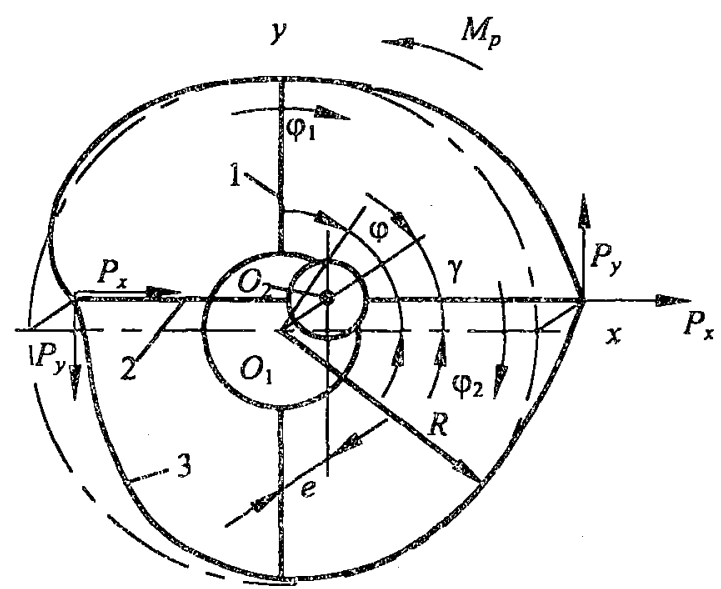

Fig 2. Deformation of the elastic ring of the coupling in case of radial misalignment we find the values of the reactions $P_{\mathrm{x}}$ and $P_{\mathrm{y}}$ as the functions of radial misalignment $e$ :

$$
\begin{aligned}
& P_{x}=\frac{2 E I}{R}(a \cos \gamma+b \sin \gamma) . \\
& P_{y^{\prime}}=\frac{2 E I}{R}(a \sin \gamma+b \cos \gamma),
\end{aligned}
$$

where

$$
a=\frac{\pi^{2}-8}{4 \pi}, \quad b=\frac{\pi-4}{2 \pi} .
$$

On the rotation of the system, an internal moment of resistance to rotation $M_{p}$ appears in the coupling that is found from the equation of moments around the point $O_{1}$ :

$$
M_{p}=2 e\left(P_{y} \cos \gamma-P_{x} \sin \gamma\right) \text {. }
$$

Using the expressions of the reactions $P_{\mathrm{x}}$ and $P_{\mathrm{y}}$ from (3), we obtain:

$$
M_{p}=157 \frac{E I}{R^{3}} e^{2} \cos 2 \gamma .
$$

In the case under discussion we may examine the rotating system as a two-mass system with two degrees of frcedom considering that its generalized coordinates are the angle of rotation of the first shaft with the halfcoupling $1 \varphi_{1}$ and the angle of rotation of the second shaft with the half-coupling $2 \varphi$, (Fig 2).

Taking into account that the angle of rotation $\gamma$ of the coupling in respect of the direction of misalignment may be interpreted as

$$
\gamma=\frac{\varphi_{1}+\varphi_{2}}{2},
$$

the expression of internal moment of resistance to rotation becomes the following:

$$
M_{p}=157 \frac{E I}{R^{3}} e^{2} \cos \left(\varphi_{1}+\varphi_{2}\right) .
$$

On the investigation of the impact of the misalignment of shafts upon the dynamics of a rotating system we suppose that rotation of the first shaft is even. in such case the generalized coordinates that describe the movement of the system will be the following:

$$
\begin{aligned}
& \varphi_{1}=\alpha_{1}+\omega t, \\
& \varphi_{2}=\alpha_{2}+\omega t+x_{e},
\end{aligned}
$$

where $\alpha_{1}, \alpha_{2}$ - the initial angles of rotation, $\omega$ - the angular speed, $t$-time, $x_{\mathrm{e}}$ - the value (angle) that characterizes the unevenness of rotation of the second shaft.

According to Dalamber's principle rotation of the second shaft is described by the following equation:

$$
I_{2} \ddot{\varphi}_{2}-M_{p}=0 \text {, }
$$


where: $I_{7}$ - the moment of inertia of the second shaft.

From the equations (7), (8) and (9), we find:

$$
\ddot{x}_{e}-157 \frac{e^{2}}{I_{2}} \frac{E I}{R^{3}} \cos \left(\alpha_{1}+\alpha_{2}+2 \omega t+x_{e}\right)=0 \text {. }
$$

We introduce a new variable:

$$
y_{e}=\frac{\pi}{2}-\left(\alpha_{1}+\alpha_{2}+2 \omega t+x_{e}\right)
$$

and find its second derivative on time and obtain the equation that virtually describes the free oscillations of mathematical pendulum:

$$
\ddot{y}_{e}+A_{e} \sin y_{e}=0
$$

where

$$
A_{e}=157 \frac{e^{2}}{I_{2}} \frac{E I}{R^{3}} .
$$

If we suppose that at the moment $t_{0} y_{e}\left(t_{0}\right)=\alpha_{y}$, $\dot{y}_{e}\left(t_{0}\right)=\beta_{y}$, we find:

$$
\dot{y}_{e}= \pm \sqrt{2 A_{e} \cos y_{g}+\beta_{y}^{2}-2 A_{e} \cos \alpha_{y}} .
$$

Then after the corresponding transformations:

$$
t-t_{0}=\int_{\alpha_{y}}^{y_{e}} \frac{d y_{e}}{\sqrt{2 A_{e}\left(\cos y_{e}-\cos \alpha_{y}\right)}+\beta_{y}^{2}} .
$$

Then the introduction of the following designations:

$$
\sin \frac{1}{2} y_{e}=k_{1} u_{1}, k_{1}^{2}=\sin ^{2} \frac{1}{2} \alpha_{y}+\frac{\beta_{y}^{2}}{4 A_{e}},
$$

the expression (14) turns into an elliptic integral of the first degree:

$$
\sqrt{A_{e}}\left(t-t_{0}\right)=\int_{\frac{1}{k_{1}} \sin \frac{\alpha_{y}}{2}}^{u_{1}} \frac{d u_{1}}{\sqrt{\left(1-u_{1}^{2}\right)\left(1-k_{1}^{2} u_{1}^{2}\right)}}
$$

that may be solved in respect of $x_{e}$.

\section{The Solution Using the Small Parameter Method}

Bearing in mind that the value of misalignment $e$ is small in comparison with other sizes and, in addition, it is raised to the second power in the expression, we'll try to find a solution of the expression (12) using the small parameter method:

$$
y_{e}=y_{e_{0}}+\varepsilon y_{e_{1}}+\varepsilon^{2} y_{e_{2}}+\ldots+\varepsilon^{n} y_{e_{n}}+\ldots \text {, }
$$

where $\varepsilon$ - a small positive parameter.

If we limit ourselves with two first members, we obtain:

$$
\left\{\begin{array}{l}
\ddot{y}_{e_{0}}=0 \\
\ddot{y}_{e_{1}}+A_{e} \sin y_{e_{0}}=0
\end{array}\right.
$$

Taking into account the initial conditions from the expression (11), we find the solutions of the system:

$$
\left\{\begin{array}{l}
y_{e_{0}}=2 \omega t+\delta_{0}, \\
y_{e_{1}}=\frac{1}{4 \omega^{2}} A_{e} \sin (2 \omega t+\delta)+2 \omega t+\delta_{1},
\end{array}\right.
$$

where $\delta_{0}, \delta_{1}-$ constants.

After the insertion of the expression (19) into the expression (17) and usage of the expression (11), the generalized coordinate $\varphi_{2}$, describing rotation of the second shaft will be equal to:

$$
\varphi_{2}=\alpha_{2}+\omega \imath+\frac{39,25 e^{2} E I}{\omega^{2} I_{2} R^{3}} \sin (2 \omega t+\delta) .
$$

The last member of the expression (20) indicates that the rotation of the second shaft is uneven, its angle of rotation is supplemented with a periodic component of the double frequency of rotation (Fig 3).

Another very important circumstance: the amplitude of this periodical part decreases increasing the angular rotational speed ( $\omega_{2}$ is included into the denominator). This indicates an increase of the evenness of the movement.

\section{The Restrictions of the Applications of the Small Parameter Method}

The presence of the angular rotational speed in the denominator of the expression of the amplitude indicates

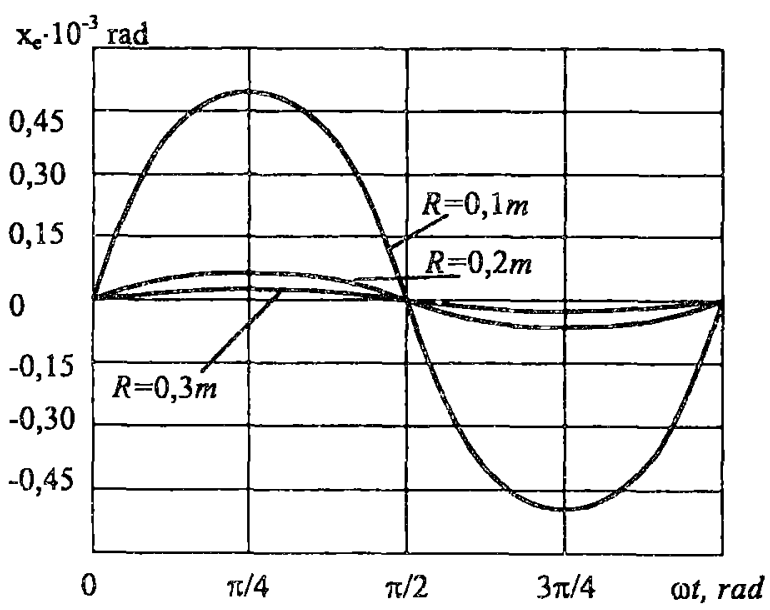

Fig 3. The dependence of the amplitude of rotational vibrations of the second shaft on the angular rotational speed

when the diameter of the wire of the ring $d=3 \mathrm{~mm}$, $I_{2}=0,05 \mathrm{kgm}^{2}, e=3 \mathrm{~mm}, \omega=100 \mathrm{~s}^{-1}, \alpha_{2}=0, \delta_{0}=0$ 
that the small parameter method cannot be applied for a solution of the equation (12) in all cases (when $\omega \rightarrow 0$, the amplitude is growing up to the infinity).

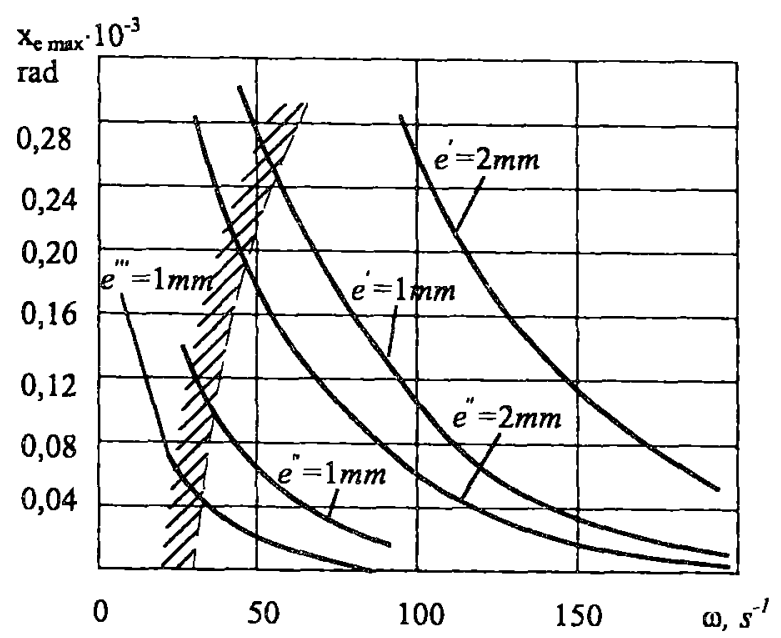

Fig 4. The dependence of the amplitude of rotational vibrations of the second shaft on the angular rotational speed, when the diameter of the wire of the ring $d=3 \mathrm{~mm},{ }^{\prime}-R=0,1$

$$
\mathrm{m}, \text { " - } R=0,2 \mathrm{~m}, \text { " }-R=0,3 \mathrm{~m}
$$

The problem on the application of the expression (20) may be settled on a base of the following considerations. The positive angle of shifting of the half-couplings in respect of each other upon an impact of the moment of resistance to rotation $M_{p}$ may be found from the following expression:

$$
x_{e}=\frac{M_{p}}{c_{s}},
$$

where $c_{s}$ - the rotational stiffness of the coupling.

$$
x_{e_{\max }}=1,8\left(\begin{array}{c}
e \\
R
\end{array}\right)^{2} .
$$$$
\text { Knowing that } c_{s}=87 \frac{E l}{R^{3}}[6] \text {, we find: }
$$

So, on the comparison of the expressions (22) and (20) according to the condition of non-exceeding $x_{\text {emax }}$, we find the lower limit value of the angular speed applicable to the expression (20) (the stroked zone in Fig 4).

\section{Conclusions}

1. Elastic centrifugal ring couplings may be successfully used for the connection of misligned shafts.

2. The rotation of rotating system misligned in radial direction may be described with the equation that virtually coincides with the equation describing free oscillations of a mathematical pendulum.

3. The frequency of the additional periodical component of the coordinate of unevenness of rotation is equal to the double frequency of the rotation of the system.

4. The application of the small parameter system is not fit in all cases. It is necessary to take into consideration the specific features of the coupling construction.

5. If two misligned shafts are connected with an elastic centrifugal ring coupling, the evenness of the rotation increases with the increase of the rotational speed.

\section{References}

1. Elbeheiry E. M. Suboptimal Bilinear Control Methods Applied to Suppesing Car Vibrations. Journal of Vibrations and Control, 2001, 7, p 279-306.

2. Muszynska. Vibrational Diagnostics of Rotating Mashinery Malfunctions. Transport Engineering International Journal of Rotating Machinery, USA, 1995, Vol 1, No 3-4, p $237-$ 266.

3. Xue S.; Tobita J.; Kurita S.; Izumi M. Mechanics and Dynamics of Intelligent Passive Vibration Control System. Journal of Engineering Mechanics, April 1997, p 322-330.

4. Turla V. Damping of torsional vibrations in motocar transmission. Transport Engineering (Transportas), Vol XVI, No 5, 2001, Vilnius: Technika, p 187-190 (in Lithuanian).

5. Spruogis B. Devices for transmission and stabilisation of rotating motion (Устройства для передачи и стабилизации вращательного движения). Vilnius: Technika, 1997. 476 p (in Russian).

6. Ragulskis K.; Spruogis B.; Ragulskis M. Transformation of rotational motion by inertia coupling. Monograph. Vilnius: Technika, 1999. $236 \mathrm{p}$. 